\title{
Oregon Bee Atlas: native bee findings from 2018
}

Lincoln Best ${ }^{1^{*}}$, Cody Feuerborn ${ }^{1}$, Jennifer Holt ${ }^{1}$, Sarah Kincaid ${ }^{1}$, Christopher J. Marshall ${ }^{2}$, Andony Melathopoulos ${ }^{1}$, Samuel V.J. Robinson ${ }^{3}$

*Corresponding author: Lincoln.Best@oregonstate.edu

${ }^{1}$ Department of Horticulture, Oregon State University, 4017 Agricultural and Life Science Building Corvallis, Oregon, USA 97331-7304

${ }^{2}$ Department of Integrative Biology, Oregon State University 3029 Cordley Hall, Corvallis, Oregon, USA 97331-7304

${ }^{3}$ Postdoctoral Associate, Department of Biological Sciences, University of Calgary, Calgary, Alberta, Canada

Cite this work as:

Best, L., C. Feuerborn, J. Holt, S. Kincaid, C.J. Marshall, A. Melathopolous and S.V.J. Robinson. 2021. Oregon Bee Atlas: native bee findings from 2018. Catalog of the Oregon State Arthropod Collection. 5 (1) 1- 12. DOI: https:/ / doi.org/10.5399/osu/cat_osac.5.1.4647.

\section{Abstract}

The Oregon Bee Atlas is a new volunteer-led effort to characterize the bee fauna of the state of Oregon by collecting, preparing, and databasing native bee species and capturing plant host records. In 2018, volunteers collected 11,044 bee specimens across 33 Oregon counties, representing 179 unique bee species, and 32 unique bee genera. Specimens were collected from a total of 310 unique flowering plant genera, resulting in one of the largest state-level databases of bee-host plant interactions. Volunteers produced valuable occurrence records for species poorly known for the State, and species of conservation concern. The 2018 efforts constitute a proof-of-concept of a specimen-focused volunteer native bee survey.

\section{Introduction}

The Oregon Bee Atlas (OBA) is a volunteer-focused initiative to survey the bee fauna of Oregon. The mission of the OBA is to train and equip citizen scientists to: (a) create and maintain a comprehensive, high quality and publicly accessible inventory of the state's native bees and their plant-host preferences, (b) to educate Oregonians on the state's bee biodiversity, and (c) to conduct an on-going survey of native bee populations in order to assess their health. Started in 2018 by Oregon State University (OSU), Oregon Department of Agriculture (ODA), and Oregon Department of Forestry $(\mathrm{ODF})$, the OBA was established as a response to the general lack of knowledge about the status of Oregon's native bees.

The state of Oregon, located in the Pacific Northwest (PNW) of the United States, presents unique challenges for detecting changes in native bee populations. These challenges stem from the fact that the State contains 12 distinct ecoregions (Level III, Environmental Protection Agency) that are dissected by two broad mountain ranges, resulting in distinct pollinator communities. Although a number of outstanding hymenopterists and pollination biologists have worked on PNW bees, their efforts were primarily based on particular groups of interest and/or specific crops. A general interest in native pollinators and their role in agriculture and ecology in more recent years has kindled an interest and need to examine the regional bee fauna as a whole. Early faunistic work on Western North American bees centered more heavily on California and other Southwestern states (see: Michener 1979, p. 238). While Moldenke (1976) compared the bee fauna of Pacific North America 
to that of Chile, that work lacks a comprehensive list of species and any means to identify them. Since the 1980's Oregon has lagged behind other parts of the US in terms of sampling efforts, with only limited and restricted studies having taken place, many of which were focused primarily on managed lands and/ or specific crops (Stephen et al. 2009; Broussard et al. 2011; Kimoto et al. 2012; Gonzalez et al. 2013; McIver and Erickson 2012; Mciver et al. 2009; Roof et al. 2018; Tubbesing et al. 2014). Publicly available records on the Global Biodiversity Information Facility show $34 \%$ more bee species occurrence records generated east of the 100th Meridian than west of it since 1980. (GBIF.org 2020). With a few exceptions (e.g., bumblebees), the PNW, and Oregon in particular, lack synoptic identification tools or even a solid authoritative checklist for its regional bees. As a result, it can be difficult, if not impossible, to reliably identify even some common taxa.

The OBA came about as a direct response to this problem, providing a unique, cost effective pathway to generate museum-quality bee specimens from areas that have historically been poorly sampled. Unlike similar programs in the United States, the OBA uses a largely volunteer workforce to collect and prepare specimens, and to provide entry level taxonomic support. Through the framework of the OBA, volunteers receive the necessary training to complete these tasks, and are partnered with taxonomists and database managers to ensure quality records are created and maintained. All observational records generated by the OBA are based on identified specimens. Exemplar specimens are deposited in the Oregon State Arthropod Collection (OSAC) to facilitate identification into the future, and so they can be used as comparative reference material. All records are made publicly available.

In addition to generating quality specimens and vouchered observational records, the OBA places a strong emphasis on community engagement and education. On an annual basis, each OBA member is required to attend at least one regional outreach event per year, with many attending multiple events. This allows OBA members to share project findings and provide information on Oregon bees and the problems they face with the general public.

Here, we present the findings from the first year of the Oregon Bee Atlas (2018), reviewing the spatial distribution of sampling, the species detected, and notable native bee discoveries.

\section{Methods}

\section{Volunteers}

Volunteer recruitment for the OBA has largely drawn from pre-existing groups, such as OSU Master Gardeners, Master Naturalists, and local beekeepers. Efforts were made to advertise training events to these audiences and the general public through Extension partnerships, outreach events, social media, and newspaper articles. Volunteer applicants were placed in teams based on proximity to training locations. Six Oregon events, which trained a total of 153 students, were held in the cities of Klamath Falls, Pheonix, Grants Pass, Canby, Corvallis, Bend, and Hood River. Trainings took place between June 2017 and February 2018, and in most instances training events lasted one full day. Topics covered included: project background, bee biology, insect collection, specimen preparation, and data entry. Two additional five-day courses were held on July 9-13 and 16-20, 2018 that covered genus-level identification of native bees in Oregon, and species-level identification of bumble bees. 
Two types of sampling were utilized by volunteers: (1) undirected, free sampling, and (2) directed sampling. For undirected sampling, volunteers were encouraged to collect bees often and wherever they chose, either independently or as a team. In directed sampling, volunteer teams were asked to target two types of sites, one with a high level of native flowering plant species, and a second site that was highly managed or disturbed, such as an intensive agricultural or highly urbanized location. Teams were encouraged to visit each site roughly every two weeks to observe seasonal variability within bee communities. Bees were primarily collected using a variable transect aerial netting, with a small subset collected using blue vane traps (see Westphal et al. 2008 for description of each method). Volunteers employing aerial netting were encouraged to collect bees from the same host plants into separate killing jars, in order to associate the bees with the host plant. In all cases, however, all sampling conducted within a 1 ha radius of where sampling started was pooled together into a common sample (e.g., aerial netting from a given plant host species growing within a 1 ha area were pooled). All bees caught from a collector within this 1 ha unit, at a given date and time, and where applicable, from a given plant, were provided with a unique identifying number (Sample ID).

\section{Observational data entry, specimen preparation, and labeling}

Volunteers recorded the following core data: (a) full name of collector, (b) a Sample ID identifier, (c) the date and time when sampling was initiated, (d) the name of the closest geographic or civic landmark to the sampling area, (e) latitude and longitude coordinates in degree decimal format, and (f) the number of bees collected. For volunteers installing passive traps, the date and time that traps were removed was also included. When netting was conducted from a specific host plant, the family, genus, and species of the host plant was recorded.

Volunteer data were recorded using two methods based on volunteer preference. A small number of volunteers used a hand-written field notebook. The majority of OBA volunteers, however, used a mobile phone in conjunction with the software package iNaturalist (www.iNaturalist.org). These volunteers were instructed to photograph and geotag an example of each plant host species that they sampled from, within a given collection area. The image was associated with the iNaturalist Project (Oregon Bee Project Regional Teams), allowing additional core data fields to be filled out by volunteers. Volunteers then returned home and pinned their specimens, ensuring that each specimen had a temporary label with the sample identification number (Sample ID). Specimen information was either: (a) extracted from the iNaturalist Project and reconfigured to a spreasheet using a Python computer script, (b) input by volunteers or, in a limited number of cases, (c) manually input by Oregon Bee Atlas staff. All data were compiled in a single spreadsheet by Oregon Bee Atlas staff at Oregon State University, who also checked each data line for errors, and contacted volunteers for clarification when incorrect or ambiguous data were discovered. Records that lacked core data fields (collector name, time and date of collection, location information) were deleted from the final dataset.

To ensure standardized and archival labels were placed on all specimens, the final labels were generated at Oregon State University on acid free cardstock paper, and then sent to volunteers to place on specimens. Label information was pulled from the verified and corrected database using a Microsoft Word Mail Merge template and then fed into templates that printed labels on 8.5" x 11" cardstock. These labels were then sent back to volunteers, who were able to place them on the appropriate specimens via their unique field notebook number ([collector name] 18.[SampleID number].[specimennumber]; e.g., LincolnBest18.21.3), which was included on the label itself. 
Once specimens were labelled, volunteers worked to provisionally identify and sort their material. They were encouraged to bring material to the Oregon Bee Atlas for monthly open microscope sessions (September-December, 2018) at Oregon State University for assistance with genus-level identification. Volunteers were instructed to turn their collections into Oregon State University by December 21, 2018 for authoritative specimen identification and voucher selection.

Specimen determinations were made by one of the authors (Lincoln Best), an experienced taxonomist with the western North American bee fauna. Individual volunteer collections were graded by Best, based on accuracy of the identification (where applicable), organization, and the skill of specimen preparation. Every volunteer who submitted a collection received an individual evaluation grading sheet. Voucher material that covered both the taxonomic and geographic breadth of the sampling was accessioned into the Oregon State Arthropod Collection, and excess material was then used to populate species-level reference collections of regional relevance for the volunteer teams.

\section{Data and metadata}

Once the annual data were collated into a single table, the data and metadata were checked for typographical errors and formatted to conform, wherever possible, to basic darwinCore biodiversity data standards (https: / / dwc.tdwg.org/). The dataset will be made publically available via the OSAC IPT server (https: / / osac.oregonstate.edu/IPT) as well as provided as a .csv text file available as a download (Supplement 1).

The final dataset contains 35 fields:

occurenceID: this represents a unique identification number for observational records. OccurrenceID is cast differently when specimens are retained. For occurrence records based on specimens housed in the collection, the occurrenceID is given the prefix: http: / / osac.oregonstate.edu / followed by 'SP / OSAC_' followed by the 10 digit museum-issued catalog number for the voucher specimens. For example: http:/ / osac.oregonstate.edu /SP /OSAC_1234567890. These URL's serve as a hyperlink to access the museum specimen record. Observations for which the basisofRecord are not retained, are issued a URL with the same prefix, however, the "SP/OSAC_XXXXXXXXXXX" is replaced with "OBS/ OBA_"+ [collectorname]+ ":" + [OBAfieldnumber]. These URLs will also retrieve the observation record, but are not tied into the museum's specimen-based inventory. An example includes: http:/ / osac.oregonstate.edu/OBS/OBA_Stephanie_Hazen:18.035.057.

catalogNumber: a unique catalog number given to a vouchered specimen for an observation deposited into the OSAC, which is presented on a printed label in both arabic human readable digits and a datamatrix barcode. As with the occurrenceID, the catalogNumber is represented in the datamatrix as the URL http:/ / osac.oregonstate.edu/SP/OSAC_XXXXXXXXXX, where the X's correspond to a unique 10-digit number.

disposition: Whereas all observational records were based on physical specimens examined by a taxonomist (Lincoln Best), not all specimens were retained. Observations for which a voucher specimen was not retained (e.g., accessioned into the museum) the catalogNumber is necessarily left blank and the disposition field indicates 'not-retained' as apposed to 'confirmedPresent'.

datasetName: The dataset name "OBA_OSAC_2018" is recorded in each record, in the event that these records are combined with other observational datasets. 
basisOfRecord: all observational records in the OBA dataset are based on actual pinned specimens, although as noted, not all specimens were archived.

fieldNumber: This number string corresponds to the Oregon Bee Atlas information recorded during the initial collecting and specimen submission process. It is important to record in case a record's history needs to be examined to verify and/or modify a record.

bibliographicCitation: Each record in the dataset is provisioned with the full bibliographic citation for the dataset, enabling downstream users to cite the record explicitly. Doing so provides an easy means to access the source of the data record and attribute proper credit to the creators of those records.

license: these data are released under a creative commons license that makes them useable for non-commercial purposes with proper and appropriate attribution (eg., cited using bilbiographicCitation above. Details on the license can be found at: http:// creativecommons.org/licenses/by-nc$\mathrm{sa} / 3.0 /$

institutionCode: OSAC

ownerInstitutionCode: OSAC

rightsHolder: Oregon State University

Collecting Date: The collecting date is stored in two forms and three separate parsed fields: day (131), month (1-12), year (2018); and an eventDate text string, is included if possible for ease of using a date format where that is desirable.

locality data: locality data is stored in 6 fields: country, state, county, location, decimalLatitude, and decimalLongitude. Georeferencing was provided by the collector and was either recorded in the field during the collecting event or determined subsequently based on maps and field notes. In all cases, they are recorded in decimal degrees with an accuracy of 3 decimal places (approximately $+/-100 \mathrm{~m}$ ).

samplingProtocol: the collecting method is typically aerial net, where other means were used: eg., sweep net or pan trap, these were indicated.

associated plant information: any bees collected in direct association with known plants has this information recorded in one of two ways. If a bee, or bees, were collected directly from a plant while foraging, this is placed into the "associatedTaxa" field, using standard language recommended by DarwinCore, in which the type of relationship is stated followed by a colon and then the plant taxon, eg., "foraging on" : "Ericameria nauseosa". In cases where a sweep net or pan trap or bees were netted in the vicinity of known plants (e.g., bees were not directly observed visiting floral resources on these plants), this is placed in fieldNotes.

recordedBy: provides the collector's name

phylum, class, order, family, subfamily, tribe, genus, species, and scientificName; all names were crosschecked against GBIF's species name tool prior to being uploaded. The full binomial with authority and date of publication is provided in scientificName. 
rank: lowest taxonomic rank the record is identified to. Sex and caste are recorded if known. repository: The repository is considered OSAC if voucher material was deposited in the museum. For observations for which the voucher was not retained, this field is left blank.

identifiedBy and dateIdentified: determination information is provided in the identifiedBy and dateIdentified fields. In all but a few cases, the final 2018 dataset records were determined by Lincoln R. Best.

\section{Results}

\section{Volunteers}

A total of 52 volunteers submitted collections of bees. Of the 52 volunteers, approximately $60 \%$ submitted collections over 50 bees, $11 \%$ submitted collections of more than 500 bees, and two volunteers submitted collections containing over 1,200 bees (Figure 1). Volunteers were largely based in counties directly west or east of the Cascade Mountains, with no collectors based in counties in Eastern Oregon (Figure 2).

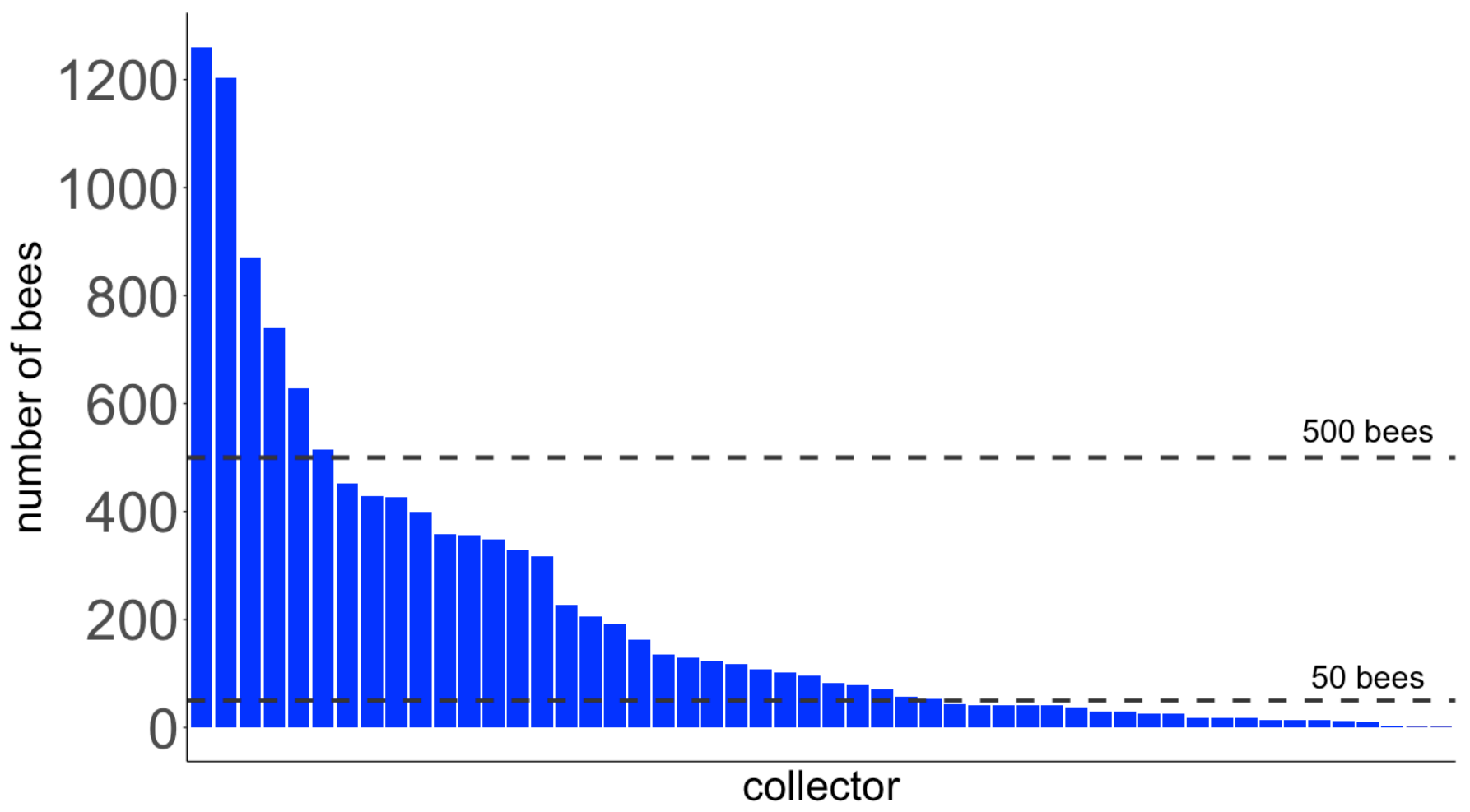

Figure 1. The number of bee specimens submitted per volunteer collector in 2018. Reference lines indicate 50 and 500 bee specimen levels. 


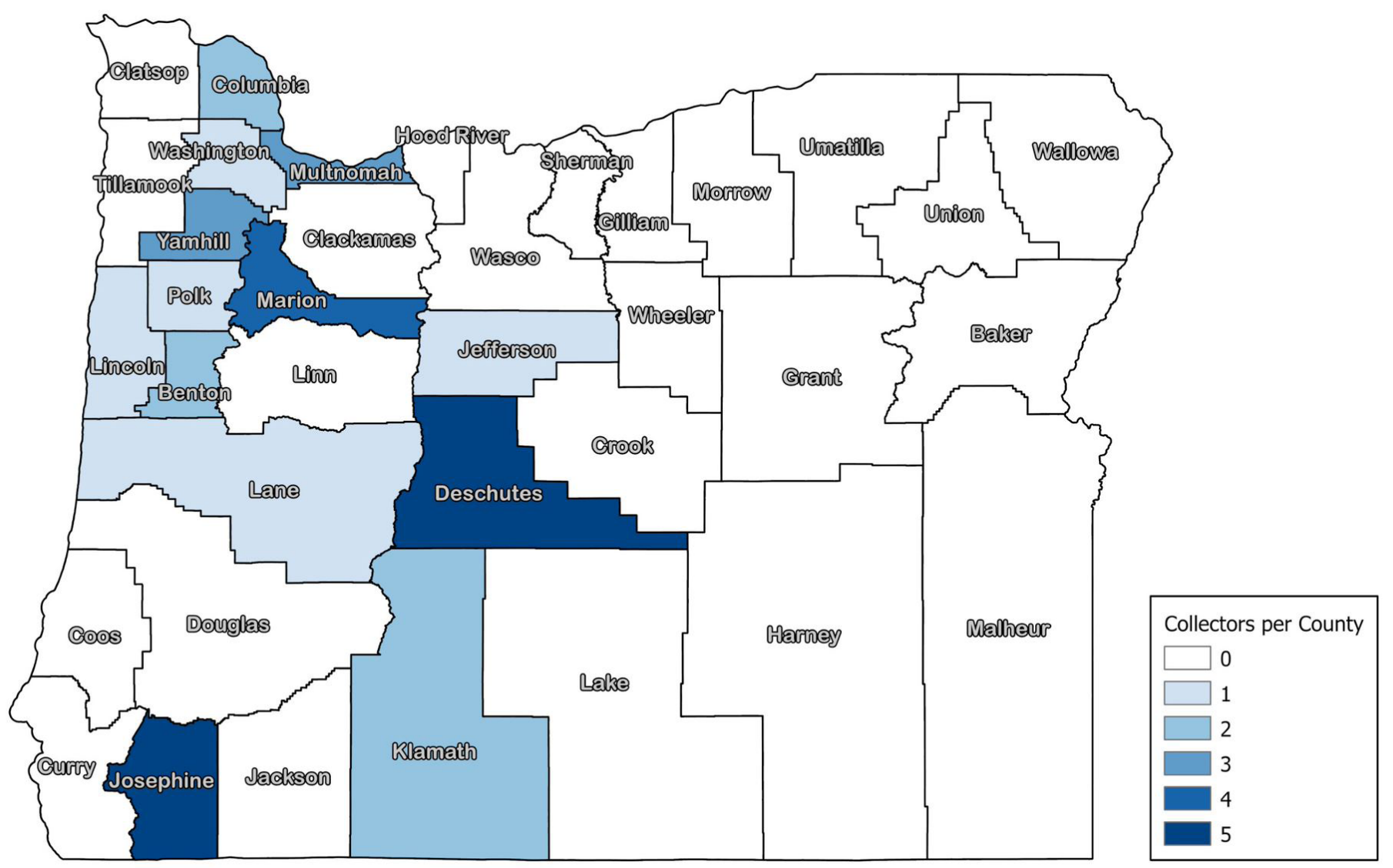

Figure 2. Distribution of where volunteers who submitted collections of more than 50 bee specimens were based in 2018. The color of each county indicates the number of volunteers based in the county.

\section{Spatial distribution of sampling}

Native bee specimens were collected from 34 of the 36 Oregon counties (Figure 3). Although specimen collection was spatially uneven across the State, $98.94 \%$ of specimens were collected outside of the Portland Metro area where most of our collectors live. Moreover, $36 \%$ of specimens were collected east of the Cascade Mountain range.

\section{Description of the native bee data}

Volunteers collected 11,044 bees across 33 counties from March 15, 2017 to August 19, 2019. Bees were predominantly collected from three bee families, Apidae, Halictidae, and Megachilidae (Figure 4). In total, volunteers collected bees from 43 genera, with the most commonly encountered genera baased on specimens being Halictus Latreille (1,906 specimens), Bombus Latreille (1,477 specimens), Osmia Panzer (1,092 specimens), Andrena Fabricius (991 specimens), and Ceratina Latreille (957 specimens). There are 179 species listed in the dataset although many specimens from a number of species-rich genera (e.g., Andrena Fabricius, Melissodes Latreille and Lasioglossum Curtis) were only determined to the generic level at the time of publication. We estimate that, once these are identified, the 2018 surveys may ultimately yield examples of over 400 species. 


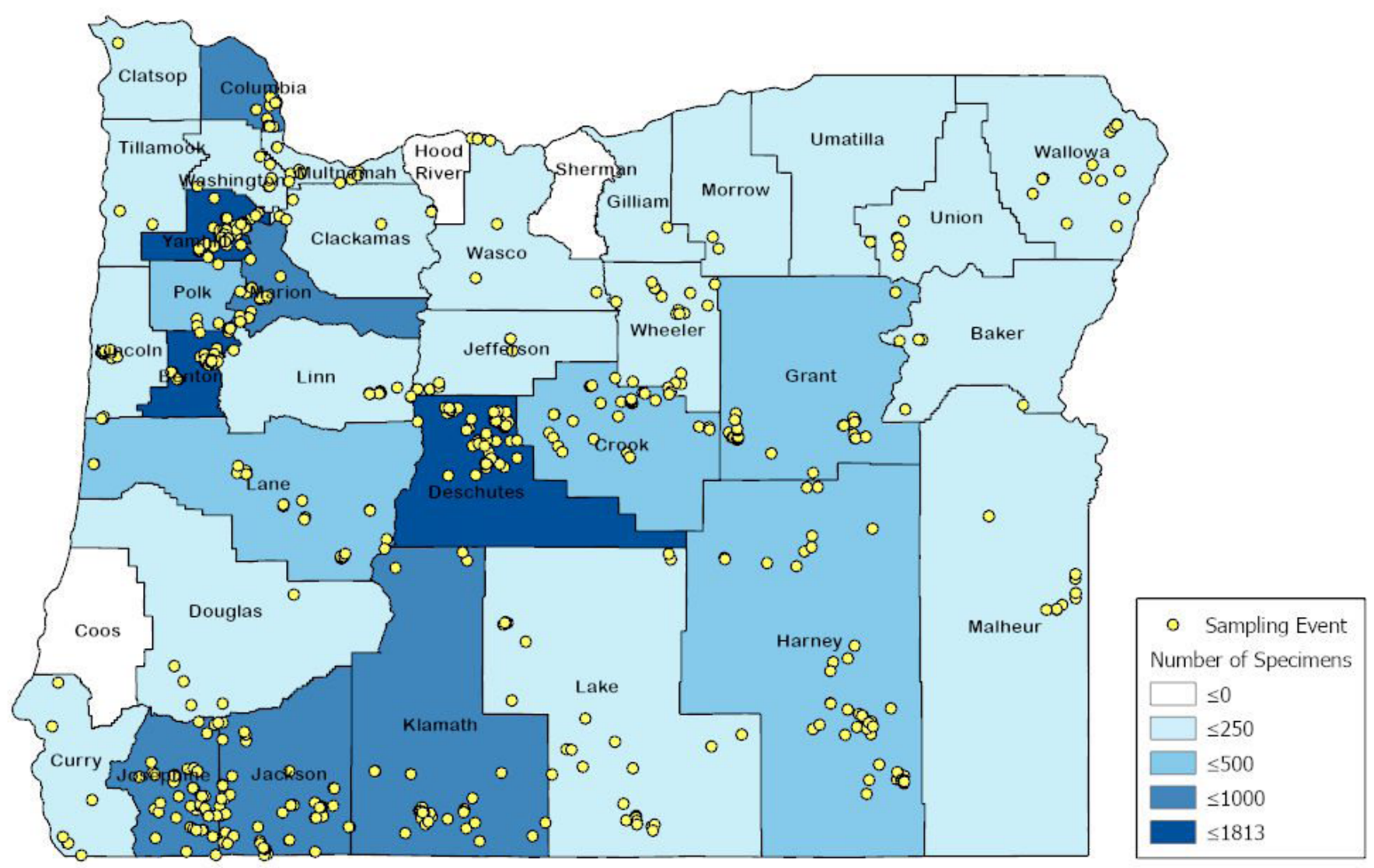

Figure 3. Heat map of 2018 volunteer sampling effort. Each dot represents a distinct sample event, which is a distinct collection within a specific bounded 1 ha area on a given date and time. The coloring of the county indicates the total number of specimens collected in the county.

\section{Notable native bee discoveries}

This state-wide survey effort produced valuable occurrence records for species poorly known for the region and for species of conservation concern. Among the Halictidae, Agapostemon melliventris Cresson was recorded from the Alvord Desert, and Halictus virgatellus Cockerell from the alpine in the Wallowa Mountains. Among the Megachilidae, Ashmeadiella (Arogochila) foxiella Michener, Ashmeadiella (Arogochila) timberlakei Michener, and Ashmeadiella (Ashmeadiella) foveata Michener were recorded from the high desert, with $A$. foveata being a novel state species record. The sand dune specialist Anthidium palliventre Cresson, was recorded from Newport, Oregon, and the rare Hoplitis (Proteriades) plagiostoma Michener was collected on Steens Mountain. In the alpine lava fields of McKenzie Pass, topotype specimens of Atoposmia oregona (Michener) were collected from its floral host Penstemon davidsonii Greene for the first time in Oregon in more than 50 years. Similarly, new occurrence records for Trachusa timberlakei (Schwarz) from the Cascade Range represent the second detection in more than 80 years in Oregon. Two infrequently detected species, Megachile mellitarsis Cresson, and Hoplitis louisae (Cockerell) were recorded from several localities east of the Cascades. The apid genus Peponapis (Say) now relegated to subgeneric status in Eucera Scopoli, was documented for the first time in Oregon in 2018 (Best et al. 2019). Twenty-one species of bumble bees (Apidae: Bombus Latreille) were recorded including the species of conservation concern Bombus caliginosus (Frison), Bombus fervidus 


\section{Species}

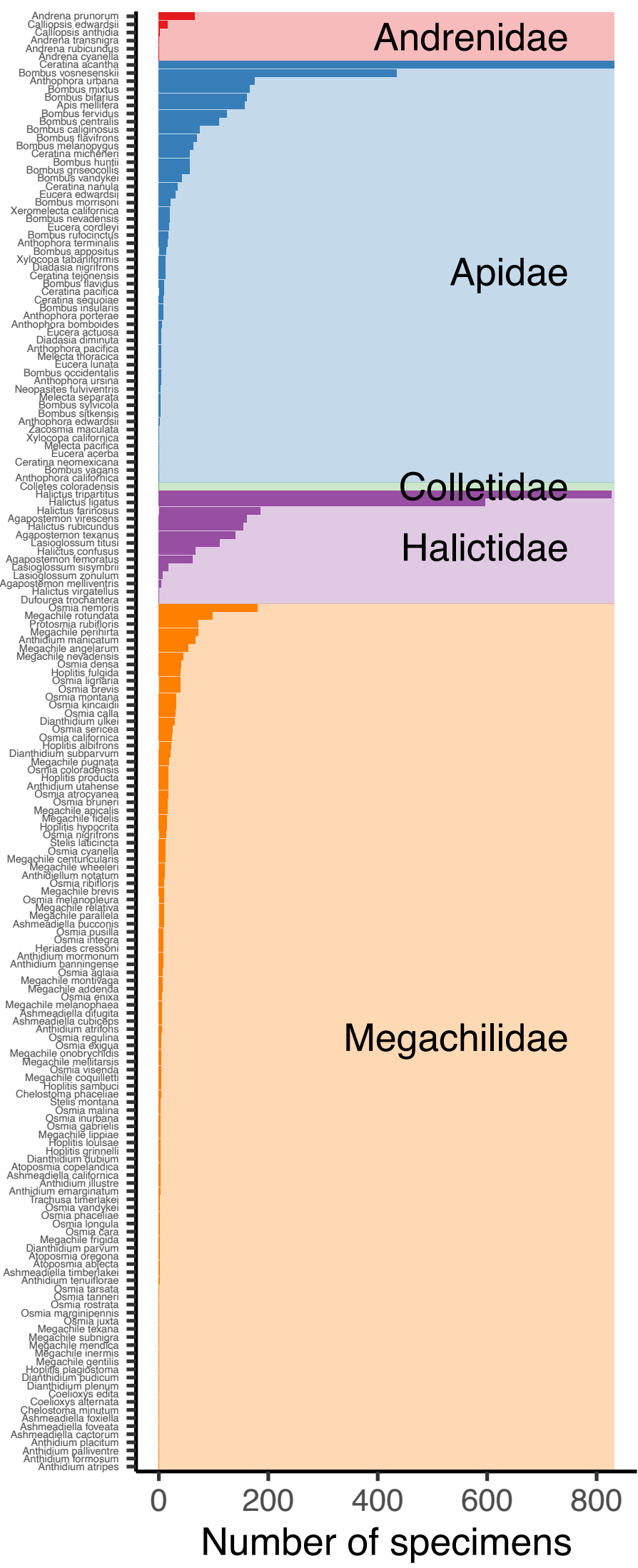

Genera

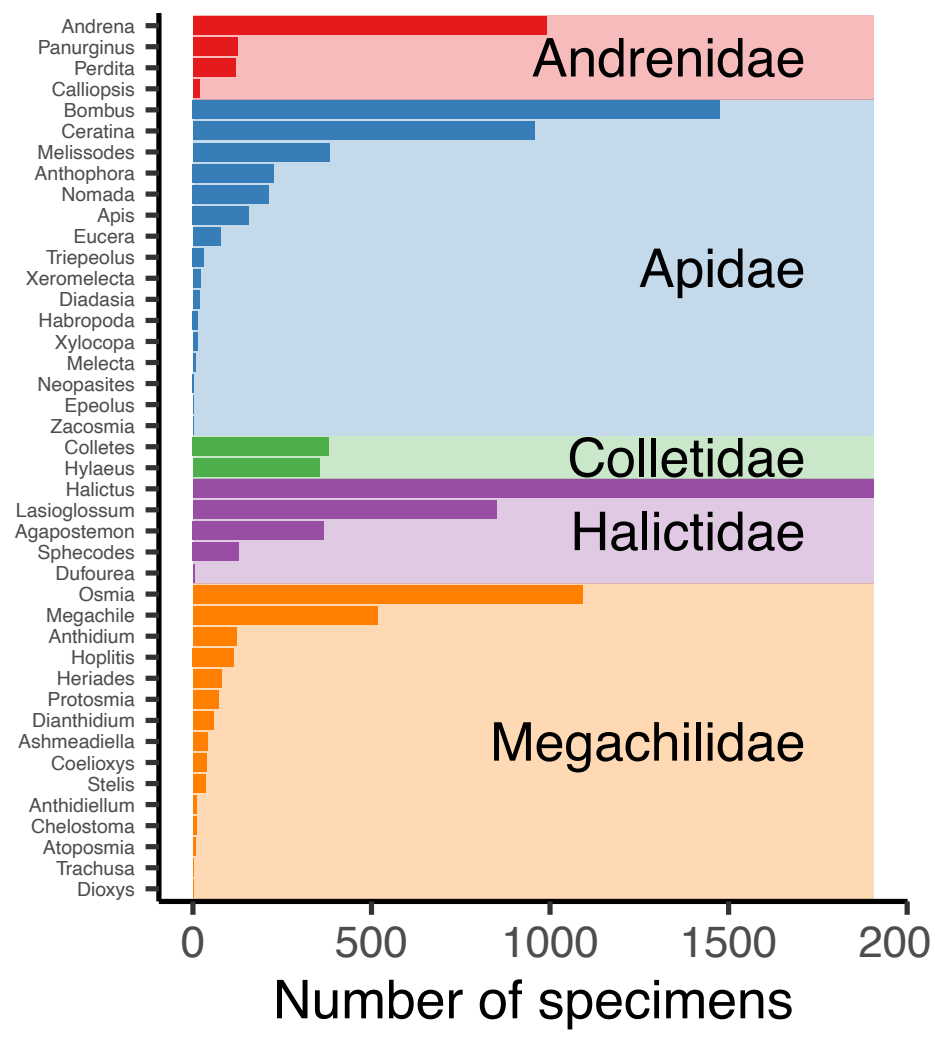

Families

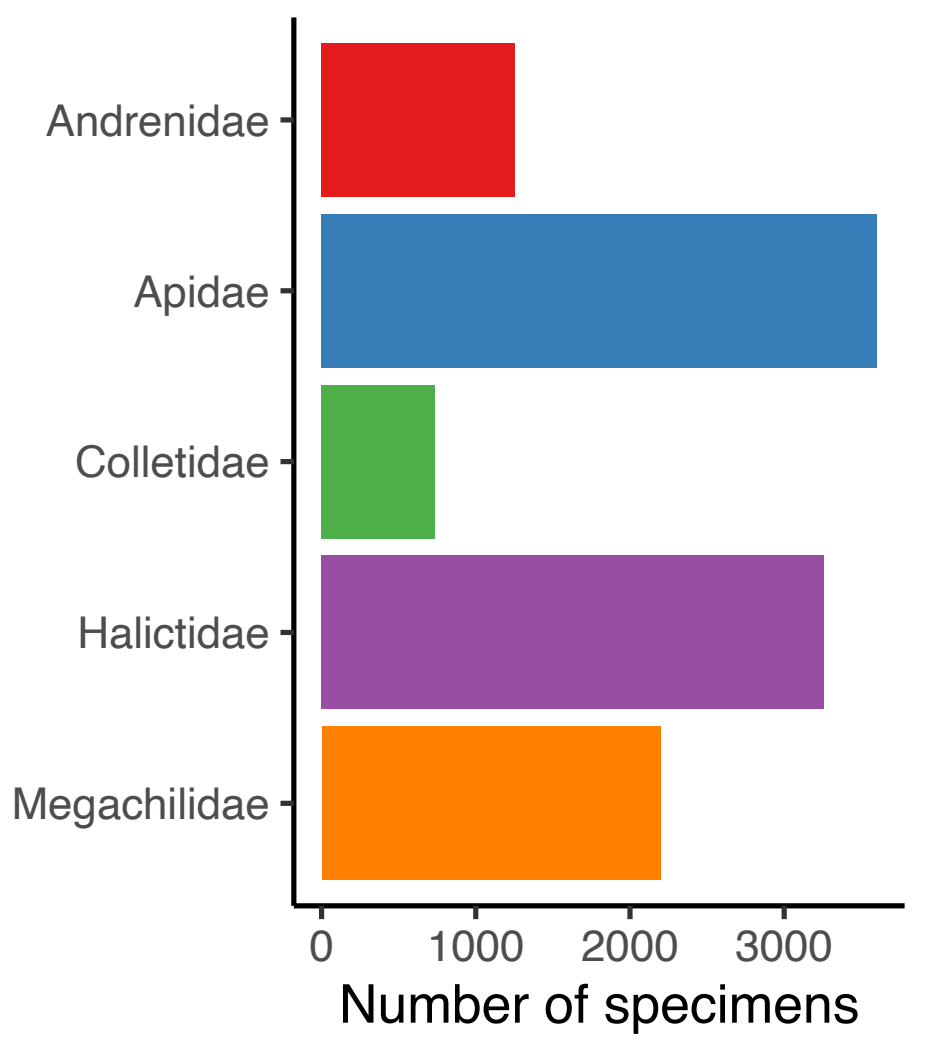

Figure 4 . Number of specimens collected in 2018 by species, genus and family. 


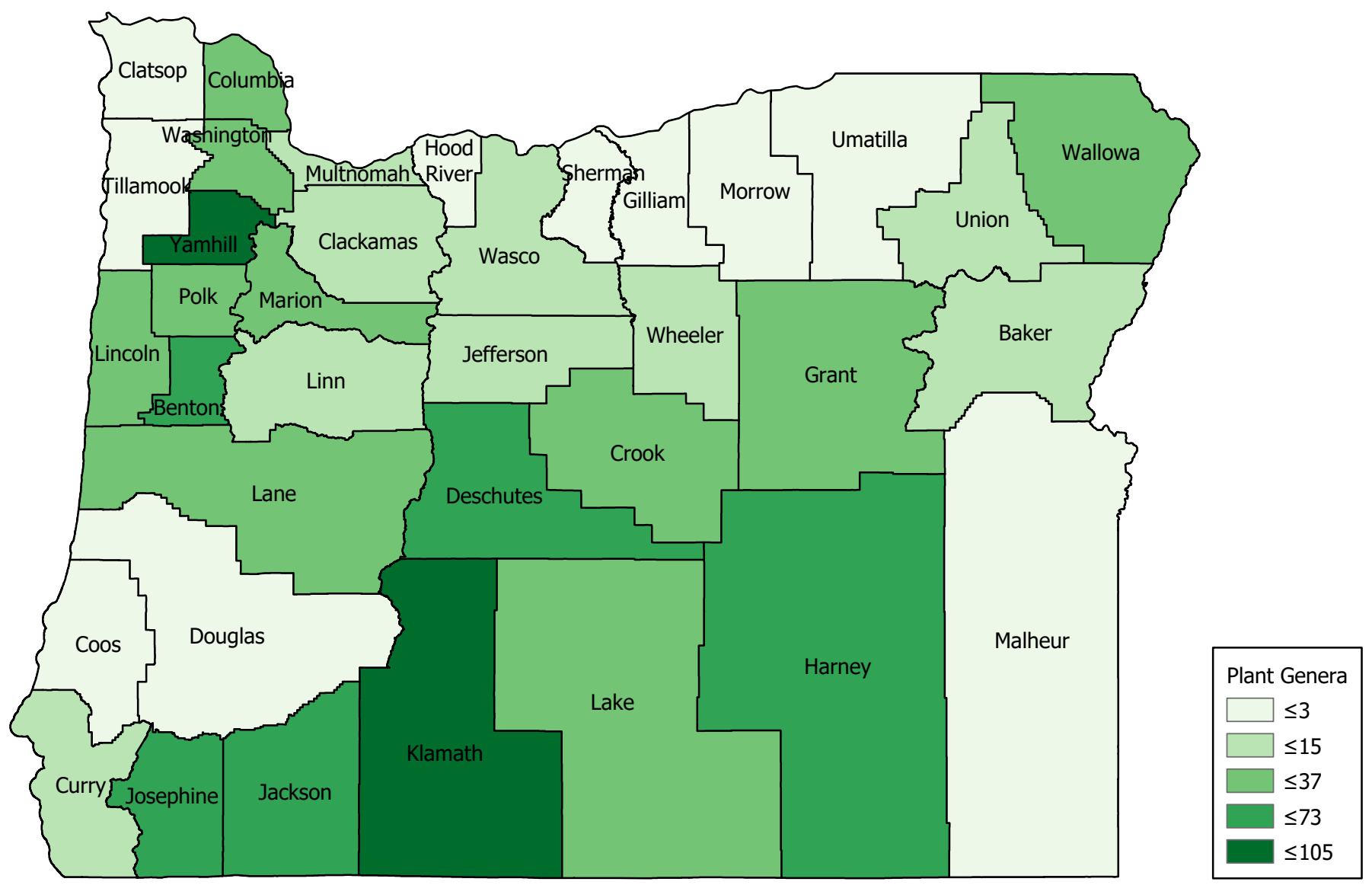

Figure 5. The number of plant genera associated with bees records in 2018, by county.

(Fabricius), Bombus morrisoni Cresson, Bombus occidentalis Greene, Bombus sylvicola Kirby, and Bombus vandykei (Frison). Bombus vagans Smith, a species uncommonly detected in Oregon was found within its known range in Wallowa County. Additional notable species records within the family Apidae were produced for Ceratina micheneri Daly, Ceratina neomexicana Cockerell, Ceratina sequoiae Michener, Ceratina tejonensis Cresson, Neopasites fulviventris (Cresson), and Zacosmia maculata (Cresson).

\section{Description of host plant data}

A total of 8,812 bee specimens were associated with a plant host (Supplement 2). The highest number of specimens were collected from Ericameria spp. (5\% of plant records), followed by Rubus spp. (3\% of plant records) and Phacelia spp. (2\% of plant records). Survey across plant genera varied considerably among counties (Figure 5). The plant genera from which the most bee species were collected were Phacelia, Rubus, and Penstemon, hosting 50, 47, and 43 unique bee species respectively.

\section{Discussion}

The first year of the Oregon Bee Atlas has demonstrated the potential of trained volunteers to provide high quality bee biodiversity data. Unlike many other volunteer-based biodiversity surveys (van der Wal et al. 2015; Dickinson et al. 2010), Atlas volunteers surveyed far beyond urban areas, sampling extensively in the sparsely populated area east of the Cascade Mountains, and locating rare species of native bees. Also, unlike most other volunteer native bee surveys where volunteers collect bee photo-vouchers (MacPhail, Gibson, Hatfield, et al. 2020; MacPhail, Gibson and Colla 2020; Falk et al. 2019; 
Richardson et al. 2019), the OBA has demonstrated the feasibility of volunteers to do more technically demanding tasks, such as collecting, preparing, and databasing specimens. Moreover, volunteers have largely collected the specimens using active survey techniques, avoiding many of the processing costs and survey biases associated with programs that rely on passive trapping of bees (Portman et al. 2020). Finally, using an innovative workflow connected to iNaturalist plant photo-vouchers, the OBA has started to build the most comprehensive bee-host plant databases of any State in the United States.

The initial results, which are biased in taxonomic identification effort towards the genus Bombus and the family Megachilidae, have provided new spatio-temporal data for many of the known species of the state fauna and have detected new state records. There are estimated more than 225 species among specimens not determined beyond the generic level, and this suggests that more than 400 species were collected throughout the state by Oregon Bee Atlas Participants in 2018. By refining floral targets and habitats, we predict that future surveys are likely to detect more than 600 species in 55 genera, including new state species, new generic records, and new data for species of conservation concern.

The Oregon Bee Atlas will continue to develop and refine its training programs to prepare volunteers to conduct more targeted surveys associated with unique plant communities in Oregon, and plans to use historical records to guide volunteers. The Atlas's short-term goal will be to develop a comprehensive checklist of Oregon native bees, including range maps. We anticipate that as our knowledge of the occurrence and distribution of native bee species increases in step with a trained cadre of volunteers, Oregon will be well positioned to join national efforts to track the long-term population trends of US bee communities.

\section{Acknowledgement}

The Oregon Bee Atlas would not be possible without the hard work and dedication of its volunteers (see Supplement 3 for a listing of volunteers who contributed to this dataset). We are particularly grateful the Oregon Bee Atlas Advisory Committee in 2018; Tom and Julie Biddle, Judi Maxwell, Michael O'Loughlin, Jerry Paul, Pete and Gretchen Peterson, Nicole Sanchez and Bonnie Shoffner. We are grateful to the Foundation for Food and Agriculture Research Pollinator Health Fund and Oregon Department of Agriculture for supporting the Oregon Bee Atlas.

\section{References}

Best, L.R., C.J. Marshall, and S. Red-Laird. 2019. Confirmed presence of the squash bee, Peponapis pruinosa (Say, 1837) in the state of Oregon and specimen-based observational records of Peponapis (Say, 1837) (Hymenoptera: Anthophila) in the Oregon State Arthropod Collection. Catalog: Oregon State Arthropod Collection.

Broussard, M., S. Rao, W.P. Stephen and L. White. 2011. Native bees, honeybees, and pollination in Oregon cranberries. HortScience 46(6), pp. 885-888.

Dickinson, J.L., B. Zuckerberg and D.N. Bonter. 2010. Citizen science as an ecological research tool: Challenges and benefits. Annual Review of Ecology, Evolution, and Systematics 41(1), pp. 149-172.

Falk, S., G. Foster, R. Comont et al. 2019. Evaluating the ability of citizen scientists to identify bumblebee (Bombus) species. Plos One 14(6), p. e0218614.

Gonzalez, N., S.J. DeBano, C. Kimoto, R.V. Taylor, C. Tubbesing and C. Strohm. 2013. Native bees 
associated with isolated aspen stands in Pacific Northwest bunchgrass prairie. Natural Areas Journal 33(4), pp. 374-383.

Kimoto, C., S.J. Debano, R.W.Thorp, S. Rao and W.P. Stephen. 2012. Investigating temporal patterns of a native bee community in a remnant North American bunchgrass prairie using blue vane traps. Journal of Insect Science (Online) 12, p. 108.

MacPhail, V.J., S.D. Gibson and S.R. Colla. 2020. Community science participants gain environmental awareness and contribute high quality data but improvements are needed: insights from Bumble Bee Watch. PeerJ 8, p. e9141.

MacPhail, V.J., S.D. Gibson, R. Hatfield and S. R. Colla. 2020. Using Bumble Bee Watch to investigate the accuracy and perception of bumble bee (Bombus spp.) identification by community scientists. PeerJ 8, p. e9412.

McIver, J. and K. Erickson. 2012. Pollination biology of Potentilla recta (sulfur cinquefoil) and Its cooccurring native congener Potentilla gracilis in Northeastern Oregon. Psyche: A Journal of Entomology 2012, pp. 1-18.

Mciver, J., R.W. Thorp and K. Erickson. 2009. Pollinators of the invasive plant, yellow starthistle (Centaurea solstitialis), in north-eastern Oregon, USA. Weed biology and management 9(2), pp. 137-145.

Michener, C.D. 1979. Biogeography of the bees. Annals of the Missouri Botanical Garden 66(3), p. 277. Moldenke, A. 1976. Evolutionary history and diversity of the bee faunas of Chile and Pacific North America. The Wasmann Journal of Biology 34, pp. 147-178.

Portman, Z.M., B. Bruninga-Socolar and D.P. Cariveau. 2020. The state of bee monitoring in the United States: A call to refocus away from bowl traps and towards more effective methods. Annals of the Entomological Society of America 113(5), pp. 337-342.

Richardson, L.L., K.P. McFarland, S. Zahendra and S. Hardy. 2019. Bumble bee (Bombus) distribution and diversity in Vermont, USA: a century of change. Journal of Insect Conservation 23(1), pp. 45-62.

Roof, S.M., S.J. DeBano, M.M. Rowland and S. Burrows. 2018. Associations between blooming plants and their bee visitors in a riparian ecosystem in eastern Oregon. Northwest Science 92(2), pp. 119-135.

Stephen, W.P., S. Rao and L. White. 2009. Abundance, diversity and foraging contribution of bumble bees to blueberry production in Western Oregon. Acta horticulturae (810), pp. 557-562.

Tubbesing, C., C. Strohm, S.J. DeBano,N. Gonzalez, C. Kimoto and R.V. Taylor. 2014. Insect visitors and pollination ecology of Spalding's Catchfly (Silene spaldingii) in the Zumwalt Prairie of northeastern Oregon. Natural Areas Journal 34(2), pp. 200-211.

van der Wal, R., H. Anderson, A. Robinson, et al. 2015. Mapping species distributions: a comparison of skilled naturalist and lay citizen science recording. Ambio 44 Suppl 4, pp. 584-600.

Westphal, C., R. Bommarco, G. Carré, E. Lamborn, N. Morison, T. Petanidou, S.G. Potts, S.P. Roberts, H. Szentgyörgyi, T. Tscheulin and B.E. Vaissière. 2008. Measuring bee diversity in different European habitats and biogeographical regions. Ecological monographs, 78(4), pp.653-671.

Williams, P. H., R.W. Thorp, L.L. Richardson, and S.R. Colla. 2014. Bumble bees of North America: an identification guide. Princeton University Press. 